\title{
Tesis de maestrías
}

\section{Docencia de la Química}

Departamento de Química, Universidad Pedagógica Nacional

\section{Modelación del enlace químico de Lewis en décimo grado de Educación Media}

Sulma Milena Urbina Duarte

\section{Descripción}

Se presenta aquí el informe final de la investigación sobre la modelación para el enlace químico propuesto por Lewis, con estudiantes del grado décimo del nivel medio del sistema educativo colombiano. Para tal efecto se acudió a la reconstrucción histórica y a la identificación y caracterización de las ideas alternativas de los estudiantes, en relación con las uniones entre átomos.

\section{Metodología}

Los estudiantes con los cuales se realiza la investigación cursan el grado décimo del nivel medio del sistema educativo colombiano en la Institución Educativa Departamental Pradilla, sede Luis Carlos Galán Sarmiento, del municipio El Colegio, Cundinamarca. Promedian los 16 años. Las preguntas centrales alrededor de las cuales gira la investigación son: ¿Hasta dónde las ideas alternativas de los estudiantes de décimo grado acerca del enlace químico se aproximan a un modelo científico?, ¿de qué manera esos modelos explicativos pueden ser punto de partida para la construcción en el aula del modelo de Lewis?

Se diseñaron y aplicaron al iniciar y al finalizar el proceso didáctico los siguientes instrumentos: prueba de composición, prueba semántica, ensayos, ejercicios de lápiz y papel y entrevista abierta, tanto para la identificación y categorización de las ideas alternativas de los estudiantes, como para la transformación de éstas y su aproximación al modelo sobre enlace químico de Lewis.

\section{Conclusiones}

De acuerdo con los resultados, empleando las diferentes pruebas, se puede concluir que con las estrategias didácticas aplicadas a los estudiantes se logró confrontar los modelos explicativos que éstos poseían, generando que en la gran mayoría, los estudiantes construyeran nuevas ideas que se aproximan al modelo de enlace químico propuesto por Kossel, Lewis y Langmuir.

Además, las ideas de los estudiantes sobre el modelo de enlace químico de Lewis, se tipificaron al iniciar y finalizar el proceso a través de la prueba semántica inicial y final, de lo cual se puede señalar que la mayoría de los estudiantes lograron una transformación satisfactoria en relación a sus concepciones acerca del modelo de enlace químico de Lewis.

Con el apoyo en la elaboración de los ensayos, los estudiantes lograron una transformación satisfactoria en relación a su concepción histórica sobre 
el modelo del enlace químico de Lewis, y se puede concluir a demás, que es necesario promover la lectura de artículos originales para acentuar que la química no es el resultado de verdades absolutas creadas por personas especiales, si no que es el resultado de un proceso y del trabajo científico.

La elaboración de los ensayos es una de las actividades que mayor dificultad presenta, debido tal vez a que en la enseñanza tradicional de la química no se enseña a leer y a escribir, limitándose únicamente a la transmisión verbal del conocimiento. Ésta es una tarea importante a realizar para empezar a desarrollar las competencias argumentativas e interpretativas en nuestros estudiantes.

Los estudiantes presentan mayor grado de aceptación por la realización de ejercicios de lápiz y papel que por la lectura de los artículos, tal vez por ser la forma más tradicional de enseñanza de las ciencias, remitida a la transmisión oral de la información; pero al finalizar, expresan su agrado por haber conocido el proceso para la construcción de este conocimiento, observándose a la vez un cambio positivo en la actitud hacia la lectura de artículos.

Las ideas iniciales de los estudiantes de décimo grado sobre el modelo de enlace químico propuesto por Lewis, aunque tratan de recordar y expresar lo construido durante los años académicos anteriores, solo en un pequeño porcentaje se aproximan a las ideas admitidas por la comunidad científica. Al finalizar el proceso, se observa que las ideas de la mayoría de los estudiantes se aproximan a las ideas admitidas por la comunidad científica, aunque la gran mayoría de los estudiantes (90\%) expresa que existe una fuerza que atrae los electrones sin describir la naturaleza de éstas para el enlace iónico y covalente.

La introducción de las estructuras de Lewis en la enseñanza es de gran importancia, pues facilita la explicación del enlace covalente, pero se presenta dificultad para explicar la unión de iones en el enlace iónico, ya que los estudiantes no tienen claras las características de las sustancias que forman cada clase de enlace.

La enseñanza de la química, a partir del conocimiento del desarrollo histórico de conceptos, por medio de la lectura de artículos originales, es una alternativa diferente y apropiada mediante la cual el proceso de enseñanza-aprendizaje de las ciencias, ya no se concibe como un proceso memorístico, sino como una estrategia motivadora y enriquecedora para el estudiante. Esta estrategia debe ser complementada, además, con un tiempo de reflexión y argumentación dentro del grupo, lo que permite al estudiante comunicar sus ideas y dudas y confrontarlas con los resultados y las argumentaciones de sus compañeros.

La reconstrucción histórica adelantada, a partir del análisis crítico de la bibliografía de los originales disponibles, constituye un aceptable recurso didáctico para que los profesores aproximen a los estudiantes, en general, a la historia de los conceptos químicos, y en particular, al modelo de enlace químico propuesto por Kossel, Lewis y Langmuir. $\Delta$ 


\title{
Un programa guía de actividades con hipermedia como apoyo didáctico para el aprendizaje significativo del enlace químico
}

\author{
William Galvis Ferrucho
}

\section{Contenido}

El presente documento se divide en cinco capítulos. El primero hace referencia al problema de investigación y contiene aspectos relacionados como los objetivos, la evaluación del problema y los límites de la investigación. El segundo contiene el marco teórico que guía la investigación, el cual contiene las directrices pedagógicas desde el ámbito sociológico, epistemológico, psicológico y de aplicación de las nuevas tecnologías; finalmente se establece la hipótesis de trabajo y el sistema de indicadores. El tercero contiene la metodología que está integrada por la muestra a investigar, la descripción de los instrumentos y el procesamiento de los datos. El cuarto enseña los datos obtenidos mediante los instrumentos con su correspondiente análisis, así como el seguimiento realizado en el aula de clases. En la quinta parte se muestran las conclusiones obtenidas mediante el trabajo de investigación.

\section{Metodología}

La estrategia metodológica empleada fue un programa guía de actividades, el cual está articulado en cinco ejes temáticos: las propiedades periódicas de los elementos químicos; el carácter iónico, covalente o metálico de las sustancias químicas; cómo influyen los electrones en la formación del enlace químico; la visión moderna del enlace químico; las formas moleculares.

Cada uno de los ejes temáticos contiene actividades de inicio, desarrollo y finalización, que tienen como hilo conductor el concepto del enlace químico. El PGA contiene como apoyo didáctico un software que le da el carácter de hipermedia, tendiente a desarrollar modelos interactivos alternativos que faciliten en los estudiantes la construcción de representaciones mentales de los fenómenos químicos asociados al enlace químico.

\section{Conclusiones}

Se establece a partir de los objetivos y la hipótesis propuesta para la presente investigación, la cual afirmó: "El diseño de un programa guía de actividades con hipermedia posibilita un aprendizaje significativo en lo conceptual, metodológico y actitudinal del concepto de enlace químico, a la luz del modelo didáctico de EAPI".

Los resultados de la prueba general revelan un cambio conceptual en los estudiantes, que se manifiesta cuando se comparan los resultados de la prueba diagnóstica en relación a la prueba final. El margen de los resultados en cada uno de los ejes temáticos propuestos señala los avances que presentaron la mayoría de los estudiantes debido a la modificación de su estructura cognitiva, lo cual se ve reflejado en sus formas de significar con relación al enlace químico. Se debe dejar constancia de que la mayor dificultad según lo señalan los instrumentos empleados radica en la comprensión de las teorías modernas del enlace químico, debido al cambio de paradigma que se 
presenta, al explicar este concepto desde la mecánica cuántica.

El aprendizaje significativo en torno al enlace químico fue posible, ya que se partió de los conocimientos que tenía cada uno de los estudiantes y, mediante la aplicación de los ejes temáticos del PGA, se fueron presentando de manera gradual nuevos conceptos de mayor poder explicativo, lo cual permitió la generación de nuevos significados que se podían relacionar con los ya existentes en su estructura cognitiva. Lo anterior se evidencia cuando se pasa de un paradigma del enlace químico representado por las estructuras de Lewis a otro de mayor poder explicativo visto desde la mecánica cuántica como lo son: la teoría del enlace de valencia y la teoría de los orbitales moleculares.

Los modelos empleados de hipermedia ayudaron a que los estudiantes realizaran construcciones imaginarias para representar diferentes fenómenos asociados al enlace químico, como la formación de moléculas, la simetría molecular, la correlación entre orbitales atómicos y moleculares, la interpretación de las curvas energéticas, entre otros.

El cambio metodológico se evidencia en los seguimientos realizados en el aula de clase, laboratorio y sala de sistemas. Los indicadores propuestos se verifican con el trabajo de los estudiantes en lo que se relaciona con el posicionamiento epistemológico mediante el análisis de lecturas relacionadas con la historia del enlace químico, la capacidad de argumentación por medio de la resolución de ejercicios justificando la respuesta, el poder de interpretación a través de los significados conseguidos en los modelos trabajados en las diferentes animaciones, y la naturaleza del trabajo científico expresado en el planteamiento de hipótesis y la conducente verificación de las mismas.

El cambio actitudinal en los estudiantes se manifiesta con el seguimiento de su trabajo en el aula, el cual se ve representado en el interés y perseverancia en el desarrollo de las actividades, el entusiasmo mostrado en el trabajo de laboratorio y la actitud positiva que asumen cuando utilizan el computador para la clase de química. Adicionalmente se pudo constatar con los resultados obtenidos en la prueba Likert que los estudiantes al finalizar el proceso pedagógico y didáctico, presentaron una actitud positiva hacia la química y su aprendizaje, que se ve reflejado en el ascenso a la categoría dos, propuesta para esta prueba.

Con relación al segundo objetivo general de la investigación, la propuesta desarrollada, de utilizar un software educativo como herramienta de apoyo en el aula de clase, tiene implicaciones en lo que hace referencia al carácter público de conocimiento que se desea fomentar, por tal razón, al escoger una plataforma que permite realizar una publicación del trabajo desarrollado en páginas web se incentiva una cultura basada en la permanente comunicación entre profesionales de la química (una comunidad), es decir, se puede utilizar la Internet como medio para convocar, discutir y realizar críticas constructivas que permitan una retroalimentación y permanente comunicación. $\boldsymbol{\Delta}$ 


\title{
Interrelaciones entre historia, epistemología y didáctica de las ciencias: el caso del modelo del Operón Lac en biología molecular. Un análisis de textos universitarios.
}

\author{
Julio Alejandro Castro Moreno
}

\section{Descripción}

Este trabajo consistió, en primer lugar, en una reconstrucción histórico-epistemológica del modelo del Operón Lac, el cual es un aspecto crucial en el desarrollo de la biología molecular y que fue construido por los científicos franceses Jacob y Monod, entre las décadas de 1950 y 1960 . Con base en lo reportado en el marco teórico y dicho estudio, se analizaron 10 textos universitarios de diferentes áreas temáticas en donde se trata el modelo en mención. El análisis propició ciertos hallazgos, los que se categorizaron en dos grupos: naturaleza de la ciencia y didactización del contenido. De acuerdo con lo argumentado en las categorías, se propone un modelo que da cuenta de las interrelaciones entre historia, epistemología y didáctica de las ciencias. Finalmente se emiten unas conclusiones y unas recomendaciones para futuras investigaciones similares.

\section{Contenidos}

Los contenidos básicos de esta tesis son: el marco teórico, en donde se abordan aspectos como los antecedentes, los aspectos históricos y epistemológicos, el surgimiento de la didáctica de las ciencias, el modelo del Operón Lac y algunas relaciones entre historia, epistemología y didáctica de las ciencias; la metodología; los resultados, que tienen que ver con la reconstrucción histórico-crítica del modelo del Operón Lac; el análisis de los textos y la propuesta de un modelo que dé cuenta de las interrelaciones entre los aspectos histórico-epistemológicos y didácticos con la actividad científica.

\section{Metodología}

A partir del estudio histórico-epistemológico y el marco teórico se elaboraron unos criterios que permitieron analizar los textos, por medio de un instrumento que se diseñó para tal fin. Los hallazgos obtenidos en el análisis permitió la construcción de dos categorías y, al retomar todo lo anterior, se propuso un modelo que diera cuenta de la problemática aquí planteada. En la metodología también se ponen de presente las preguntas e hipótesis de investigación, los libros seleccionados y las razones de por qué se escogieron y, finalmente, la fases por las que transitó la investigación

\section{Conclusiones}

El estudio histórico permitió comprender algunos aspectos de la actividad científica, recurriendo a los artículos originales y a otros escritos hechos por los autores del modelo en cuestión. De otro lado, se puede concluir que en la elaboración de los textos universitarios, al parecer, no se tienen en cuenta los aspectos mencionados y, por tanto, propiciarían la transmisión de una visión tergiversada del quehacer científico. Finalmente, como en el análisis hecho no se da cuenta de las interrelaciones objeto de problema, fue pertinente proponer un modelo que las haga explícitas. $\Delta$ 


\title{
Maestrías en Tecnologías de la Información Aplicadas a la Educación
}

\section{Resolución de problemas apoyada en tecnologías de la información y de comunicación para el aprendizaje significativo de la geometría estructural en la química del carbono}

\author{
Alfonso Raúl Trujillo Campos
}

\section{Descripción}

La presente investigación tiene por objetivo desarrollar una alternativa de innovación educativa que articule los programas guía de actividades, la resolución de problemas de lápiz y papel, el trabajo práctico de laboratorio con el apoyo de las TIC, para el mejoramiento de la comprensión de la hibridación de los orbitales del átomo de carbono, la percepción espacial de sus moléculas y sus formas de representación. Para conseguirlo se plantea el diseño y elaboración de un programa de actividades interactivo que pueda ser desarrollado en el aula escolar (PAIA) en tiempo real, o pueda ser implementado vía Internet en tiempo no lineal. El tipo de investigación utilizada es descriptiva correlacional y busca relacionar la incidencia de estos nuevos PAIA con el aprendizaje de los conceptos de hibridación y su influencia en las formas de representación geométricas de las moléculas. Como resultado se produce un PAIA y un cambio significativo en las formas de representación molecular por parte de los estudiantes que tiene alcances hasta en el diseño digital de las mismas. Quedan planteadas las inmensas posibilidades de la transformación de los programas de actividades como unidades didácticas interactivas que posean la visión de una enseñanza globalizada vía Internet.

\section{Contenidos}

Introducción: resumen de la tesis desde los PGA a los PAIA. Justificación: unificación de los PGA con apoyo de las TIC para el aprendizaje significativo. Antecedentes teóricos: revisión histórico-epistemológica del concepto de hibridación orbital. Fundamentación pedagógica: teoría del aprendizaje significativo de Ausubel. Fundamentación didáctica. Uso de los PGA en enseñanza por investigación escolar. Fundamentación desde las TIC: uso de visualizadores, animaciones, software, web. Antecedentes empíricos: enseñanza por investigación escolar y uso de TIC en el aula. Planteamiento del problema: diseño y aplicación del PAIA en los estudiantes para A. S. Planteamiento de la hipótesis: diseñar y aplicar PAIA. Establecer relaciones entre el programa y el aprendizaje significativo del concepto de hibridación y geometría molecular.

Definición operacional de la hipótesis: diseño, forma de aplicación del PAIA. Objetivos. Desarrollo de PAIA. Establecimiento de relaciones entre PAIA y aprendizaje. Método. Investigación descriptiva correlacional transeccional. Resultados. Producción de un PAIA. Descripción de la relación entre el programa y el aprendizaje de concepto, formas de representación y asimilación de los mismos. Discusión: propuesta exploratoria 
del uso de PAIA, aprendizaje significativo representacional y de las formas geométricas de las moléculas.

\section{Metodología}

Enmarcado en un tipo de investigación descriptiva correlacional transeccional como un estudio que tiene por propósito evaluar la relación que exista entre dos o más conceptos, categorías o variables de un contexto particular. Busca explorar mediante observación sistemática el tipo de relaciones entre el aprendizaje significativo del concepto de hibridación de orbitales atómicos y las formas geométricas de las moléculas por medio de un PAIA, que puede ser usado en el aula o por Internet. Se elabora una rejilla de observación por actividad en donde aparecen los aspectos a ser observados y evaluados por estudiante en cada actividad. Se realizó análisis de resultados y conteo de frecuencias con respecto al resultado observado en los estudiantes, mediante una escala cualitativa que establecía el valor de los argumentos y justificaciones ante cada actividad.

\section{Conclusiones}

Desarrolla en los estudiantes aprendizaje de representaciones como el uso de términos precisos para la nomenclatura de las moléculas, diferenciación en los conceptos de tipos de isomería y una clara explicitación de los principios en la teoría estructural y luego la asimilación del concepto de hibridación como explicación de la formación de los diferentes tipos de enlace y de sus consecuencias sobre la geometría espacial de las moléculas; por tanto, se muestra el aprendizaje de conceptos en el manejo de modelos de representación para las moléculas tanto análogos como digitales, uso de la nomenclatura orgánica y entendimiento de ésta. Queda planteado como trabajo exploratorio que propone la posible evolución del los PGA y la enseñanza en un contexto globalizado marcado claramente por el uso de las TIC por parte de la comunidad educativa. $\boldsymbol{\Delta}$

\title{
Estrategias de aprendizaje en razonamiento bidimensional. Geometría de las transformaciones
}

\author{
Dora Almáriz Rueda Velásquez
}

\section{Descripción}

La presente tesis pretende desarrollar software educativo basado en la representación de dominios de conocimiento mediante sistema de ontología, que potencie el desarrollo y la evaluación del aprendizaje significativo. Además, identificar aquellos procesos cognitivos presentes en los procedimientos que los alumnos ejecutan en la solución de problemas sobre transformaciones en el plano, teniendo en cuenta seis dimensiones: pensamiento sistémico, manejo de información, síntesis, pensamiento crítico, conceptualización y análisis. Es- 
tudiar las estrategias para el desarrollo de habilidades del pensamiento abstracto, en particular en el campo del razonamiento bidimensional. Es importante usar el computador como herramienta para acceder a variadas dimensiones de comprensión y a su vez promover la creatividad de los estudiantes en ambientes virtuales.

\section{Contenido}

Capítulo primero: marco teórico. Se da la visión conceptual y teórica de conceptos como hipertexto, metacognición, aprendizaje significativo, activadores de juicios de metamemoria y las consideraciones pedagógicas y didácticas que dieron origen a este proyecto. Capítulo segundo: antecedentes. Se hace un recorrido por las investigaciones más recientes tanto a nivel tecnológico como del tema específico a tratar. Capítulo tres: dominio de conocimiento. Se hace un análisis de la representación del conocimiento y sus posibilidades, tema que sirvió no solo para la creación del hipertexto basado en una ontología, sino para ver en los mapas conceptuales un sistema propicio para la evaluación de los objetivos propuestos. Además, se desarrollan temas como cognición y matemáticas, relación entre aprendizaje y conducta y por supuesto nuestro tema: transformaciones en el plano.

Capítulo cuatro: metodología. Se presentan las especificaciones del hipertexto a utilizar, de los ambientes experimentales, el diseño de los activadores de metamemoria, y descripción de todos los pasos seguidos en el presente proyecto, enmarcados en la pregunta de investigación, las hipótesis y el sistema de variables. Capítulo cinco: análisis estadístico de datos. Se hace un análisis de los datos obtenidos para establecer la relación entre las variables: activadores de juicios de metamemoria en diferentes tiempos y la comprensión de conceptos. Capítulo seis: interpretación de resultados, realizada con base en cuatro momentos, la afirmación basada en los resultados (a la luz de la obtención de respuestas a situaciones problémicas), la evidencia y la asociación con el marco teórico y el aporte específico. Capítulo siete: conclusiones. Se integran los resultados obtenidos y generalizan, para obtener respuestas a las preguntas de la investigación.

\section{Metodología}

La presente investigación estudia el efecto que causan sobre el aprendizaje situaciones problémicas dadas en un momento previo al uso de un hipertexto sobre transformaciones bidimensionales y cuando se entregan posterior a la exploración del mismo. Se aplica a dos grupos de séptimo grado, cada uno de 40 alumnos escogidos de manera aleatoria. Se consideran dos fases: una ejecutoria y otra de generalización. En la primera se proporciona al grupo 1 un hipertexto sobre el tema junto con siete situaciones problémicas que actúan como activadores previos, las cuales deben contestar en un lapso 160 minutos. En esta misma parte, el grupo 2 realiza primero la exploración libre del mismo hipertexto, y luego recibe las mismas preguntas, así podemos considerar el momento como posterior. La segunda fase o de generalización consiste en 80 mnutos en los cuales los individuos de ambos grupos contestan una prueba llamada general, que contiene seis preguntas, 
en la que se busca comparar aprendizajes días después de la primera fase. Cada situación problémica pertenece a una dimensión que corresponde a las capacidades de orden superior: análisis, síntesis, conceptualización, manejo de información, pensamiento sistémico y pensamiento crítico. Son evaluadas con 32 ítems con puntajes dependiendo del grado de acertividad. Para el análisis cuantitativo se usaron dos métodos estadísticos: prueba t para medias de dos muestras emparejadas y análisis de varianza para un factor.

\section{Conclusiones}

La presente investigación muestra que a partir de la presentación de una situación problémica, los sujetos activan las estrategias que tienen almacenadas en su memoria a largo plazo trayéndolas a su memoria de trabajo. Algunos alumnos proceden a descartar aquellas que no generan solución, mientras que otros insisten y solo las descartan después de varias pruebas. Es predecible que los sujetos intenten trabajar con lo que saben y luego traten de incorporar nuevas estrategias dependiendo de los errores cometidos, modelo expresado por Pirolli (1991). En los dos grupos las ideas se relacionan con lo que el alumno ya sabe; los nuevos conocimientos se vinculan de manera estrecha con los anteriores, esto se puede inferir teniendo en cuenta los requisitos conceptuales de cada una de las preguntas. Así, se evidencia la tesis del aprendizaje significativo que plantea Ausubel: lo aprendido se relaciona sustancialmente con la estructura cognoscitiva previa. Hay un aprendizaje del tema de transformaciones en el plano (traslación, rotación y reflexión), conclusión inferida por el resultado del trabajo en la solución de los problemas planteados para cada una de las dimensiones. Se evidencian dificultades con los conocimientos previos en ambos grupos. En el primero, la lectura y representación del problema inducen a los alumnos a examinar los contenidos del hipertexto, lo cual lleva a una revisión de conceptos previos para su comprensión total. Para el segundo grupo, después de la lectura del hipertexto, los alumnos reciben las situaciones problémicas y con base en la representación que de cada una hacen, se dan cuenta que igual, tanto los conocimientos previos como los conceptos adquiridos con el hipertexto son necesarios para la solución de los problemas.

Los problemas más complejos (en cuanto al número de elementos que intervienen en el problema, conceptos utilizados y pasos para la solución) son los más difíciles de resolver. Si en las pruebas se utilizan enunciados similares (problemas de la misma clase: de la misma complejidad, que comparten estrategias) a los de los problemas tomados como ejemplos dentro del hipertexto, los alumnos ya tienen pautas para la solución; en caso contrario tendrán que recurrir a nuevas estrategias. Atendiendo a Ausubel, Novack y Hanesian (1973, p. 145) en esta investigación se evidencia el aprendizaje autónomo, (tomado en términos de las decisiones que el alumno toma para lograr la meta). En cada paso el estudiante tiene diferentes alternativas de caminos y sea cual sea el que tome debe hacer una evaluación del proceso que lleva para decidir. Así, construye su camino de decisión y así mismo el camino está conformado por un conjunto de decisiones. 
Los individuos exploran y construyen las relaciones necesarias para interpretar y manejar las posibles soluciones a los problemas planteados. Además, enriquecen su conocimiento previo con decisiones que están bajo su control. Citando a Vannevar Bush (p. 20), el aprendizaje funciona por asociación: sujetando un hecho o una idea, "la mente salta instantáneamente al dato siguiente, que le es sugerido por asociación de ideas, siguiendo alguna intrincada trama de caminos conformada por las células del cerebro... el hipertexto nos permite seguir nuestra tendencia natural a la "selección por asociación, y no mediante los índices". Así, el hipertexto ofrece la posibilidad de facilitar que el usuario se convierta en un procesador activo: él construye su propio mapa de navegación, jerarquiza los conceptos del hipertexto y los relaciona con el problema en el momento en el que le es suministrado.

El sistema de representación ontológico, utilizado en el hipertexto, incide en la construcción conceptual de los estudiantes, que se refleja en los mapas conceptuales que deben hacer en la prueba general. Los sujetos guardan la característica de herencia entre los conceptos y subconceptos, con relaciones pertinentes entre ellos. La importancia que da George Polya (p. 64) a la pausa, la reflexión y la ejecución de pasos originales, es decir, a lo creativo en la solución, (que distingue un problema de un ejercicio), se nota en la solución a las preguntas 4 y 5 de la prueba particular, en las cuales los estudiantes utilizan varios métodos, diferentes al sugerido, como leer a la tras luz o girar $180^{\circ}$ la hoja, para hallar las respuestas a las situaciones problémicas. $\mathbf{\Delta}$

\section{Efectos de un modelo de comprensión de texto en tres niveles de complejidad dentro de un ambiente hipermedial como estrategia de intervención pedagógica en el desarrollo de habilidades de lectura en inglés}

Deisy Baracaldo Guzmán

\section{Contenidos}

El informe de investigación corresponde al esquema de trabajo de tesis. Se presenta una justificación y una revisión del estado del arte que incluye estudios alrededor del tema relacionados con experiencias alrededor de hipertextos y de modelos de lectura. En el marco de referencia se presentan la fundamentación lingüística y pragmática, el texto y la lingüística textual, el discurso y la prag- mática, las funciones del lenguaje dentro del proceso lector, las estrategias lectoras, los modelos sobre la comprensión de textos escritos, el proceso de lectura en una segunda lengua, las diferencias individuales, los cambios generacionales y el hipertexto como herramienta de enseñanza-aprendizaje. La metodología señala las dimensiones cuantitativas y cualitativas del estudio, definiendo para 
ello, las hipótesis, el diseño de investigación, la muestra y la estrategia de experimentación. Se presentan los sustentos pedagógicos y tecnológicos, la estructura del software y el procedimiento experimental con el mismo.

\section{Metodología}

Teniendo en cuenta que el objetivo de la investigación es el de revisar aspectos como la competencia para el manejo de elementos lingüísticos y la aplicación de estrategias de lectura en inglés, la investigación tiene en cuenta las dimensiones cualitativa y cuantitativa, definiendo el estudio como una investigación de tipo cuasiexperimental, utilizando para ello el diseño estadístico y la observación no estructurada. En un momento previo a la interacción con el software, se aplica el pretest que permite evaluar las estrategias aplicadas y el nivel de competencia de manejo del código lingüístico en la comprensión textual; posteriormente se lleva a cabo la experimentación en donde el grupo de estudiantes de tercer semestre del Departamento de Química, se divide en dos subgrupos con el fin de manipular el software, esto es, un grupo trabaja con el dispositivo respaldado por el modelo de lectura y el otro grupo trabaja con el mismo dispositivo pero sin modelo, es decir, pueden navegar libremente porque no hay restricciones. En un tercer momento se aplican las pruebas postest y se obtienen las observaciones después de la interacción con el dispositivo permitiendo identificar los cambios en las estrategias y en la competencia del manejo del código lingüístico después de utilizar el software; cambios presentados entre el antes y el después en cada grupo (intragrupo) y entre grupos (intergrupos).

\section{Conclusiones}

Entre otras se pueden destacar: el software es un dispositivo que motiva los procesos de lectura desde los intereses particulares de los estudiantes. Las estrategias utilizadas en los procesos de comprensión textual, cambian con la utilización del dispositivo, acercándose a las estrategias utilizadas por los mejores lectores en lengua extranjera. La competencia en el manejo del código lingüístico y en los procesos de comprensión textual presenta cambios significativos en el grupo que manejó el dispositivo respaldado por el modelo, frente a los cambios que presentaron los estudiantes que manejaron el dispositivo sin modelo, los cuales no son tan significativos si se comparan con los del grupo 1 . Se requieren futuras investigaciones en donde se realicen estudios en profundidad con el fin de abordar desde otras perspectivas las dimensiones manejadas en la comprensión lectora en esta investigación. $\Delta$ 


\title{
Los ambientes de aprendizaje una herramienta para mejorarla retención de algoritmos. Ambiente hipertextual ambiente secuencial en la retención de algoritmos.
}

\author{
José Francisco Amador Montaño
}

\section{Descripción}

Esta investigación cuenta con antecedentes que reúnen aspectos relacionados con la memoria, la representación de algoritmos mediante esquemas estáticos y dinámicos apoyados en animaciones, así como los procesos de almacenamiento de la información en la memoria humana. De otro lado el marco teórico trata especialmente de la retención significativa, el aprendizaje autónomo, los ambientes de aprendizaje para la programación y el conocimiento específico de manejo de lista en lenguaje $\mathrm{C}++$ apoyado en algoritmos y representaciones. Para el desarrollo de esta investigación se tuvieron dos grupos que interactuaron con ambientes de aprendizaje (ambiente hipertextual y ambiente secuencial) por un lapso de tiempo, después presentaron una prueba para determinar la retención a corto plazo, y pasados siete días, se les aplicó la misma prueba para determinar la retención a largo plazo. Para el procesamiento de los resultados arrojados por las dos evaluaciones en los dos momentos, se utilizó el programa Statistica.

\section{Contenidos}

El problema que trata esta investigación es la identificación de diferencias significativas en la retención de algoritmos en dos ambientes de aprendizaje. De los antecedentes de mayor incidencia en esta investigación se cuenta con las investigaciones realizadas por el grupo de investigación del Georgia Institute of Technology citado en las fuentes de esta investigación. Parte del aprendizaje autónomo, haciendo un recorrido por los ambientes de aprendizaje, la representación diagramática, los diferentes modelos teóricos de la memoria y su implicaciones en la memoria a corto y a largo plazo para concluir con el aprendizaje significativo y la retención significativa. La metodología que se abordó para esta investigación es de corte cuasiexperimental, se trabajó con dos grupos en diferentes ambientes y se hicieron dos evaluaciones en dos momentos diferentes para cada grupo. Se consideraron como variables independientes los ambientes de aprendizaje y como variable dependiente la retención de algoritmos.

\section{Metodología}

Esta investigación es cuasiexperimental, debido a que no se organizaron los grupos de manera aleatoria, sino que se organizaron de acuerdo a como estaban distribuidos por grupos asignados por la universidad donde se llevó a cabo. El desarrollo de esta investigación está compuesto por cinco estadios. Estadio 1: trata de la lectura de un texto acerca de los algoritmos de listas en lenguaje C. Esta información es similar a la que se encuentra en los libros de texto para esta 
asignatura. Estadio 2: los estudiantes se familiarizan con el uso del software, sus partes y su funcionamiento. Sobre recorrido de algoritmos a través del software y la información a la que acceden desde el comienzo de la interacción hasta el cierre de la sesión con el programa. Estadio 3: los estudiantes interactúan con el software por espacio de dos horas. Donde podrán ver los algoritmos y ejemplos, recorriendo cada una de las partes del programa. Estadio 4: los estudiantes presentan una evaluación donde se mide la retención de los algoritmos, teniendo en cuenta las variables de retención de disponibilidad, discriminalidad y estabilidad, evaluadas a través de las acciones de corregir, construir, completar e identificar algoritmos de manejo de listas en lenguaje C. Estadio 5: Los estudiantes presentan una segunda prueba a los siete días donde se aplican los mismos parámetros de evaluación que en la primera prueba.

\section{Conclusiones}

Hay mayor retención de los algoritmos de listas interactuando con el ambiente de aprendizaje hipertextual que con el ambiente de aprendizaje secuencial en los dos momentos de evaluación. Los estudiantes que interactuaron con el ambiente de aprendizaje hipertextual obtuvieron puntajes superiores en la retención de algoritmos en la primera y segunda evaluaciones que aquellos que lo hicieron con el ambiente de aprendizaje secuencial. En ambas evaluaciones la media estadística siempre es superior en el grupo del ambiente hipertextual, frente al grupo del ambiente secuencial. Hay diferencias estadísticamente significativas en la retención de algoritmos de manejo de listas en el segundo momento de evaluación, es decir, que la diferencia reside en la memoria a largo plazo, pues, se observa que aumentó la variabilidad intergrupalmente y disminuyó la variabilidad intragrupalmente. Dando como cierta la hipótesis $\mathrm{H}_{2}$ de esta investigación, es decir, hay mayor retención de algoritmos de listas en los estudiantes que interactuaron con el ambiente de aprendizaje hipertextual frente a los que lo hicieron con el secuencial.

Desde la teoría de la memoria operativa, Baddeley y Hitch (1974), el ambiente de aprendizaje hipertextual posibilitó que el ejecutivo central planificara y supervisara tanto el procesamiento de la información como del funcionamiento de los restantes sistemas para lograr una mayor retención. Desde el punto de vista de la agenda viso-espacial se concluye que el ambiente hipertextual y el ambiente secuencial propiciaron una retención creciente entre los dos momentos de evaluación debido a que los algoritmos tenían referencia directa con imágenes que representan el conocimiento de listas en $\mathrm{C}++$, lo que hace más fácil aprender y recordar. $\Delta$ 